\title{
Cryptotanshinone inhibits IgE-mediated degranulation through inhibition of spleen tyrosine kinase and tyrosine-protein kinase phosphorylation in mast cells
}

\author{
SUMIYASUREN BUYANRAVJIKH* , SORA HAN*, SUNYI LEE, AE LEE JEONG, HYE IN KA, JI YOUNG PARK, \\ ARIUNDAVAA BOLDBAATAR, JONG-SEOK LIM, MYEONG-SOK LEE and YOUNG YANG \\ Department of Biological Science, Sookmyung Women's University, Seoul 04310, Republic of Korea
}

Received December 15, 2017; Accepted May 4, 2018

DOI: $10.3892 / \mathrm{mmr} .2018 .9042$

\begin{abstract}
Atopic dermatitis (AD) is a type of chronic skin inflammation and one of the most common relapsing allergic diseases, which presents with a severe rash and itchy skin lesions. The pathogenesis of AD is primarily associated with hyper-activated mast cells, which makes them an effective treatment target. After cross-linking the antigen/immunoglobulin (Ig) E complex binds to its high affinity receptor FceRl on the surface of mast cells. The cells subsequently secrete excessive pro-inflammatory mediators, including histamine and cytokines, which lead to pruritus and immune cell infiltration in the skin lesions. The present study screened natural compounds that have an inhibitory effect on IgE/antigen-mediated secretory activity. It was revealed that cryptotanshinone (CRT), a natural compound extracted from Salvia miltiorrhiza Bunge, had inhibitory effects on the IgE/antigen complex. The underlying mechanism by which CRT exerted an anti-allergy/inflammatory function was investigated using rat basophilic leukaemia (RBL) cells for degranulation assays and a 1-chloro-2,4-dinitrobenzene (DNCB)-induced AD Balb/c mouse model for in vivo study. CRT effectively mitigated the secretion of pro-inflammatory cytokines, including tumor necrosis factor- $\alpha$ and interleukin $1 \beta$, as well as immune cell infiltration into skin lesions in a mouse model of AD-like skin disease induced by dinitrochlorobenzene. The inhibitory effect of CRT on IgE-mediated mast cell degranulation was mediated by the inhibition of tyrosine kinase-dependent degranulation signalling pathways involving spleen tyrosine kinase and Lyn. The present study
\end{abstract}

Correspondence to: Professor Young Yang, Department of Biological Science, Sookmyung Women's University, Cheongpa-ro 47-gil-100, Yongsan-gu, Seoul 04310, Republic of Korea

E-mail: yyang@sookmyung.ac.kr

${ }^{*}$ Contributed equally

Key words: atopic dermatitis, cryptotanshinone, 1-chloro-2,4-dinitrobenzene, Balb/c, degranulation, RBL-2H3 revealed CRT as an inhibitor of mast cell degranulation. Therefore, CRT may be considered for development as a therapeutic drug to treat IgE-mediated skin diseases.

\section{Introduction}

Atopic dermatitis (AD) is a chronic skin inflammation and one of the most common relapsing allergic diseases. The incidence of AD has gradually been increasing worldwide. The etiology of the disease is associated with innate and adaptive immune responses, which are caused by environmental and genetic factors; AD mostly occurs in infants and children(1). The clinical characteristics of AD are prevalently related to an excessive accumulation of antigen and imbalance of allergen-specific T helper (Th)1/Th2 cells and inflammatory cytokines. Those inflammatory cytokines contribute to increase levels of immunoglobulin (Ig) E and produce other pro-inflammatory mediators to trigger infiltration of inflammatory immune cells, such as granulocytes, lymphocytes, macrophages, eosinophils, and mast cells into skin lesions (2). The repeated exposure of antigen produces more severe and chronic AD symptoms, including skin barrier disruption, pruritus, excoriation, and dryness. In most cases, patients with AD are treated with synthetic steroids. However, many clinical reports have warned that long-term use of synthetic steroids may result in side effects such as additional infections, gastrointestinal ulcers, osteoporosis, and insomnia (3-5). Therefore, the identification of novel anti-allergic naturally-derived agents from herbs and medicinal plants with less side effects are required for AD treatment.

Mast cells play an important role in type I hypersensitivity reactions via the release of histamine, chemokines, and various inflammatory cytokines. Secretion and activation of these strong pro-inflammatory mediators are stimulated by binding of the cross-linked IgE/antigen complex and its high affinity receptor FceRl on surfaces of mast cells (6). Previous studies have revealed that IgE/antigen-FceRl binding activates I $\mathrm{B}$ kinase $\alpha$ and $\beta$ (IKK $\alpha$ and $\mathrm{IKK} \beta$ ), leading to the activation of nuclear factor $-\kappa \mathrm{B}(\mathrm{NF}-\kappa \mathrm{B})$, which translocates into nucleus to regulate the inflammatory response. Conversely, IgE/antigen-FceRl binding phosphorylates synaptosome-associated protein 23 (SNAP-23) 
in an $\mathrm{NF}-\kappa \mathrm{B}$-independent manner, which is responsible for late-phase allergic reactions $(7,8)$. Finally, activated $\mathrm{NF}-\kappa \mathrm{B}$ increases the production and secretion of pro-inflammatory cytokines including tumor necrosis factor $\alpha$ (TNF- $\alpha$ ), interleukin (IL)-1 $\beta,-4$, and -6 $(9,10)$. Accordingly, it is considered that both the inhibition of pro-inflammatory cytokine production and of $\mathrm{NF}-\kappa \mathrm{B}$ dependent signalling molecules are an effective strategies to alleviate allergic reactions.

Previous studies have revealed that tanshinones, including tanshinone-I, tanshinone-IIA, and 15,16-dihydrotanshinone-I, reduce allergic reactions in rat mast cells $\mathrm{RBL}-2 \mathrm{H} 3$ via suppressing their degranulation. More specifically, 15,16-dihydrotanshinone-I inhibits the activation of extracellular signal-regulated kinases 1/2 (ERK1/2), spleen tyrosine kinase (Syk), and phospholipase C $\gamma 2$ (PLC $\gamma 2)$ which are signalling molecules that induce mast cell degranulation (11). Furthermore, components of Salvia miltiorrhiza Bunge have anti-allergic, anti-inflammatory, and anticancer activities, $(12,13)$ and are used to treat cardiovascular disorders $(14,15)$ Cryptotanshinone (CRT), one of the major natural compounds extracted from the medicinal herb Salvia miltiorrhiza Bunge, also belongs to the tanshinone group. In addition, CRT is known as an inhibitor of signal transducer and activator of transcription 3 (STAT3). Because STAT3 is a transcription factor that increases the transcription of pro-inflammatory cytokines, CRT is also able to inhibit the production of these cytokines. Specifically, CRT strongly inhibits phosphorylation at the Tyr705 residue at STAT3 with a small effect at the Ser727 residue, but has no activity against STAT1 or STAT5 (16). To date, little has been reported regarding the precise molecular target by which CRT inhibits mast cell degranulation.

\section{Materials and methods}

Reagents. CRT, Dulbecco's modified Eagle's medium (DMEM), foetal bovine serum (FBS), phosphate-buffered saline (PBS), dinitrophenyl-bovine serum albumin (DNP-BSA), anti-dinitrophenyl IgE isotype (DNP-IgE), 4-nitrophenyl-N-acetyl-D-glucosamine, citrate buffer, sodium bicarbonate, 1-chloro-2,4-dinitrobenzene (DNCB), lipopolysaccharide (LPS), and 10\% neutral-buffered formalin were purchased from Sigma-Aldrich (Merck KGaA, Darmstadt, Germany). Dimethyl sulfoxide (DMSO) was purchased from Takara Bio Inc. (Shiga, Japan). Dexamethasone and primary antibody against $\beta$-actin were purchased from Santa Cruz Biotechnology Inc. (Dallas, TX, USA). Primary antibodies against phospho-I $\kappa \mathrm{B} \alpha, \mathrm{IkB} \alpha$, phospho-NF- $\kappa \mathrm{B}$ p65, NF-кB p65, phospho-Lyn, Lyn, phospho-Syk, Syk, phospho-PLC $\gamma 1$, PLC $\gamma 1$, phospho-protein kinase C (PKC), PKC, phospho-ERK1/2, and ERK1/2 were purchased from Cell Signaling Technology, Inc., Danvers, MA, USA.

Cell culture. The rat basophilic leukaemia (RBL) cell line RBL-2H3 was a kind gift from professor Jean-Pierre Kinet (Harvard University, Cambridge, MA, USA). The RBL-2H3 cell line shares characteristics with human mucosal mast cells (17-19), which makes it is an appropriate cell line to use within the present study. RBL-2H3 cells were cultured in DMEM supplemented with $10 \% \mathrm{FBS}$ at $37^{\circ} \mathrm{C}$ in an incubator under $5 \% \mathrm{CO}_{2}$ conditions.
Mast cell degranulation assay. RBL-2H3 cells were plated in 6 -well plate $\left(2 \times 10^{6}\right.$ cells/well $)$ or 96 -well plate $\left(5 \times 10^{4}\right.$ cells/well and were sensitized with anti-DNP-IgE $(0.1 \mu \mathrm{g} / \mathrm{ml})$ for $16 \mathrm{~h}$. After washing two times with PBS, the cells were pre-treated with CRT at indicated concentrations for indicated times then sensitized with DNP-BSA (100 ng/ml) for an additional $1 \mathrm{~h}$. For the measurement of $\beta$-hexosaminidase release (a biomarker of degranulation) from RBL-2H3 cells, $50 \mu \mathrm{l}$ of cell supernatant was incubated with a same volume of solution I [substrate solution: $1.3 \mathrm{mg} / \mathrm{ml}$ of 4-nitrophenyl-N-acetyl-D-glucosamine in $0.1 \mathrm{M}$ sodium carbonate] at $37^{\circ} \mathrm{C}$ for $1 \mathrm{~h}$ and the reaction was terminated by adding stop solution II [50 mM sodium carbonate] for $15 \mathrm{~min}$ at room temperature. The measurement of $\beta$-hexosaminidase release was determined using a microplate reader at an absorbance of $405 \mathrm{~nm}$ (Molecular Devices, LLC, Sunnyvale, CA, USA).

Luciferase assay. 293T cells were transfected with $200 \mathrm{ng}$ of pGL3-4xNF-kB luciferase reporter plasmid using polyethylenimine solution (Sigma-Aldrich; Merck KGaA), then incubated overnight. pEGFP plasmid was used as control. 5, 10, $20 \mu \mathrm{M}$ CRT was pre-treated to transfected cells for $1 \mathrm{~h}$. Then, cells were stimulated with LPS. After $1 \mathrm{~h}$ of incubation, the cells were lysed and luciferase activity was determined using Luciferase Reporter Assay System (Promega Corp., Madison, WI, USA) according to the manufacturer's instructions.

Animals. Male 6-week old Balb/c mice (20-25 g; Koatech, Gyunggi-do, Korea) were housed under 12-h light/12-h dark conditions and were allowed free access to food and water. The bedding was changed once a week, and the temperature $\left(22-23^{\circ} \mathrm{C}\right)$ and humidity $(40-55 \%)$ were controlled. All procedures were conducted at the animal facilities and this animal experiments were approved by the Institutional Animal Care and Use Committee of Sookmyung Women's University, Seoul, Korea (SMWU-IACUC-1611-035).

$D N C B$-induced $A D$ animal model. Balb/c mice were randomly divided into three groups ( $n=5$ per group). The day after shaving the dorsal skin of all mice, the control, and experimental groups were sensitized by the application of DNCB solution by painting (dissolved in a 3:1 mixture of acetone and olive oil). After 5 days, $20 \mu 1$ of $0.2 \%$ DNCB solution was applied on both the left and right ears, and $100 \mu \mathrm{l}$ was applied on the shaved dorsal skin every other day; the vehicle group received applications of DMSO only. In the experimental group, the same volume of $100 \mu \mathrm{M}$ CRT was applied by painting on both ears and on the dorsal skin $1 \mathrm{~h}$ before every DNCB challenge. The thickness of right and left ears of all mice were measured every other day with a dial caliper (Ozaki Factory, Tokyo, Japan). All mice were sacrificed on day 31 of the experiment by $\mathrm{CO}_{2}$ euthanasia and tissues were collected.

Histological analysis. The inflamed ear specimens of each mice were collected and fixed with $10 \%$ neutral-buffered formalin. All fixed tissues were embedded using a frozen section compound and were cut into $20 \mu \mathrm{m}$-thick sections using a rotary microtome (both Leica Microsystems, Inc., Buffalo Grove, IL, USA). To compare the swelling of the epidermis and inflammatory cell accumulation, each 
section was stained with haematoxylin and eosin (H\&E; Sigma-Aldrich; Merck KGaA). For immunofluorescence, tissues were treated with PBS-based $0.1 \%$ Triton-X-100 for 10 min to permeabilize the tissue. After washing with PBS, slides were blocked by PBS-based $1 \%$ BSA for $30 \mathrm{~min}$ at room temperature. Then slides were incubated with phycoerythrin (PE) conjugated-anti-mouse-cluster of differentiation molecule 11b (CD11b; CA, USA), a marker of inflammatory granulocytes for $1 \mathrm{~h}$ at room temperature in the dark. After washing with PBS twice, slides were cover-slipped. Confocal images were obtained with a Zeiss confocal microscope (Carl Zeiss Microscopy GmbH, Jena, Germany).

Measurement of IgE levels by enzyme linked immunosorbent assay (ELISA). Blood was collected by cardiac puncture from isoflurane-anesthetized mice on the last day of experiments. Clotted blood samples were centrifuged (3,500 rpm for $20 \mathrm{~min}$ ) and serum was collected. Ear tissues from mice were homogenized with RIPA buffer [20 mM Tris-HCl, $\mathrm{pH}$ 7.5, $150 \mathrm{mM} \mathrm{NaCl}, 1 \mathrm{mM}$ EDTA, 1\% NP-40, 1\% SDS, Complete Protease Inhibitor Cocktail Tablets (Roche, Basel, Switzerland)] and centrifuged at 12,000 g for $15 \mathrm{~min}$ to obtain tissue lysates. The level of $\mathrm{IgE}$ from mice serum and tissue lysates were determined by a commercial mouse IgE ELISA assay kit (Shibayagi, Shibukawa, Japan) according to the manufacturer's instructions.

Quantitative RT-PCR (qRT-PCR) Frozen tissue from mice or RBL-2H3 cells were lysed with RNAiso plus reagent (Takara Bio Inc.) and total RNA was extracted according to the manufacturer's instructions. The isolated total RNA was reverse transcribed (RT) using M-MuLV RTase (Promega Corp.) at $42^{\circ} \mathrm{C}$ for $1 \mathrm{~h}$. qRT-PCR was performed using $\mathrm{SYBR}^{\circledR}$-Green master mix (Thermo Fisher Scientific, Inc., Waltham, MA, USA) and Applied Biosystems QuantStudio 3 Real-Time PCR System (Thermo Fisher Scientific, Inc.). 18s rRNA was used as loading control Fold changes of indicated mRNA expression were calculated by the $2^{-\Delta \Delta C t}$ method, where $\Delta \Delta C \mathrm{t}=(C \mathrm{t}$ target gene- $C \mathrm{t} 18 \mathrm{~S}$ rRNA $)$. Experimental group $-(C \mathrm{t}$ Target gene- $C \mathrm{t} 18 \mathrm{~S}$ rRNA) Control group. The following primer pairs were used: IL- $1 \beta$ forward, 5'-AGCCCATCCTCTGTG ACTCATG-3' and reverse, 5'-GCTGATGTACCAGTTGGG GAAC-3'; IL-6 forward, 5'-CCGGAGAGGAGACTTCAC AG-3' and reverse, 5'-TCCACGATTTCCCAGAGAAC-3'; TNF- $\alpha$ forward, 5'-CCTGTAGCCCACGTCGTAGC-3' and reverse, 5'-TTGACCTCAGCGCTGAGTTG-3'; monocyte chemoattractant protein 1 (MCP-1) forward, 5'-ATCCCAATG AGTAGGCTGGA-3' and reverse, 5'-CAGAAGTGCTTGAGG TGGTT-3'; 18s rRNA forward, 5'-AGCTATCAATCTGTC AATCCTGTC-3', and reverse, 5'-GCTTAATTGACTCAACAC GGGA-3'.

Western blot analysis. Cells and tissue from mice were lysed with GST-IP buffer [50 mM Tris-HCl, pH 7.5, $150 \mathrm{mM} \mathrm{NaCl}$, 1 mM EDTA, $0.5 \%$ NP-40, Complete Protease Inhibitor Cocktail Tablets (Roche)] or RIPA buffer, respectively. For analysing phosphorylated proteins, PhosSTOP EASYpack (Roche) was added to the lysis buffer. Protein lysates were obtained by further centrifuge (13,000 rpm for $15 \mathrm{~min})$. Then, obtained lysates were mixed with 5x sodium dodecyl sulphate (SDS) sample buffer and heated at $95^{\circ} \mathrm{C}$ for $5 \mathrm{~min}$. Prepared sample were separated by $12 \%$ SDS-Polyacrylamide gel and transferred to a nitrocellulose membrane (GE Healthcare, Little Chalfont, UK). After blocking with TBST-based $3 \% \mathrm{BSA}$ for $30 \mathrm{~min}$ at room temperature, the membranes were incubated with indicated primary antibodies at $4^{\circ} \mathrm{C}$ overnight. Then, membranes were further incubated with horseradish peroxidase (HRP)-conjugated anti-mouse or anti-rabbit IgG (Fab) secondary antibodies (Enzo Life Sciences Inc., Farmingdale, NY, USA) for $2 \mathrm{~h}$ at room temperature. The target proteins were analysed by PowerOpti-ECL western blotting reagent (Thermo Fisher Scientific, Inc.) and evaluated using a luminescent image analyser Fusion Solo (Vilber Lourmat, Eberhardzell, Germany). The size of protein in each blot was expected by relative migration ratio to prestained protein size marker (Thermo Fisher Scientific, Inc.) and $\beta$-actin in each blot was detected to normalize protein amount. Images were quantified using ImageJ software (National Institutes of Health, Bethesda, MD, USA).

Statistical analysis. All the data were expressed as the mean \pm standard deviation (SD). All multiple comparisons within groups were made with either one-way or two-way factorial ANOVA. Significances were determined with Tukey's honest significant difference post hoc test. Individual group mean differences were determined with Student's t-test. A maximum level of significance of $\mathrm{P}<0.05$ was used for all statistical comparisons. All statistical analyses were performed using GraphPad Prism version 5.0 for Windows (GraphPad Software, Inc., La Jolla, CA, USA).

\section{Results}

CRT suppressed IgE-mediated mast cell degranulation. In order to identify effective candidates having anti-allergic activity, we screened 133 natural compounds with an IgE-mediated degranulation assay using RBL cell line RBL-2H3, which is widely accepted and applied as a proper model for human mucosal mast cells. Compared to other compounds, CRT showed the greatest anti-allergy effects as it significantly decreased IgE/DNP crosslink-mediated degranulation in RBL-2H3 cells (Fig. 1A). Furthermore, CRT suppressed IgE/DNP-mediated mast cell degranulation dose-dependently and its effects at the maximum dose was similar to that of dexamethasone at $10 \mu \mathrm{g} / \mathrm{ml}$ (Fig. 1B). No cytotoxic effects of CRT were observed (Fig. 1C), indicating that the suppressive effect of CRT on mast cell degranulation was not due to cytotoxicity.

CRT alleviated DNCB-induced AD symptoms in the mouse model. To determine whether CRT has curable effects on AD-like skin lesions, a DNCB-induced AD animal model was generated using Balb/c mice and was then subjected to CRT treatment as shown in the experimental design (Fig. 2). The repeated DNCB challenge successfully induced AD-like symptoms, as mice showed markedly increased ear swelling as compared to the vehicle group. When CRT was pre-treated $1 \mathrm{~h}$ before every DNCB challenge, ear swelling was significantly reduced after 2 weeks of CRT treatment (Fig. 3A and B). Histological analyses also confirmed that CRT treatment attenuated AD-like inflammation and skin tissue damage 

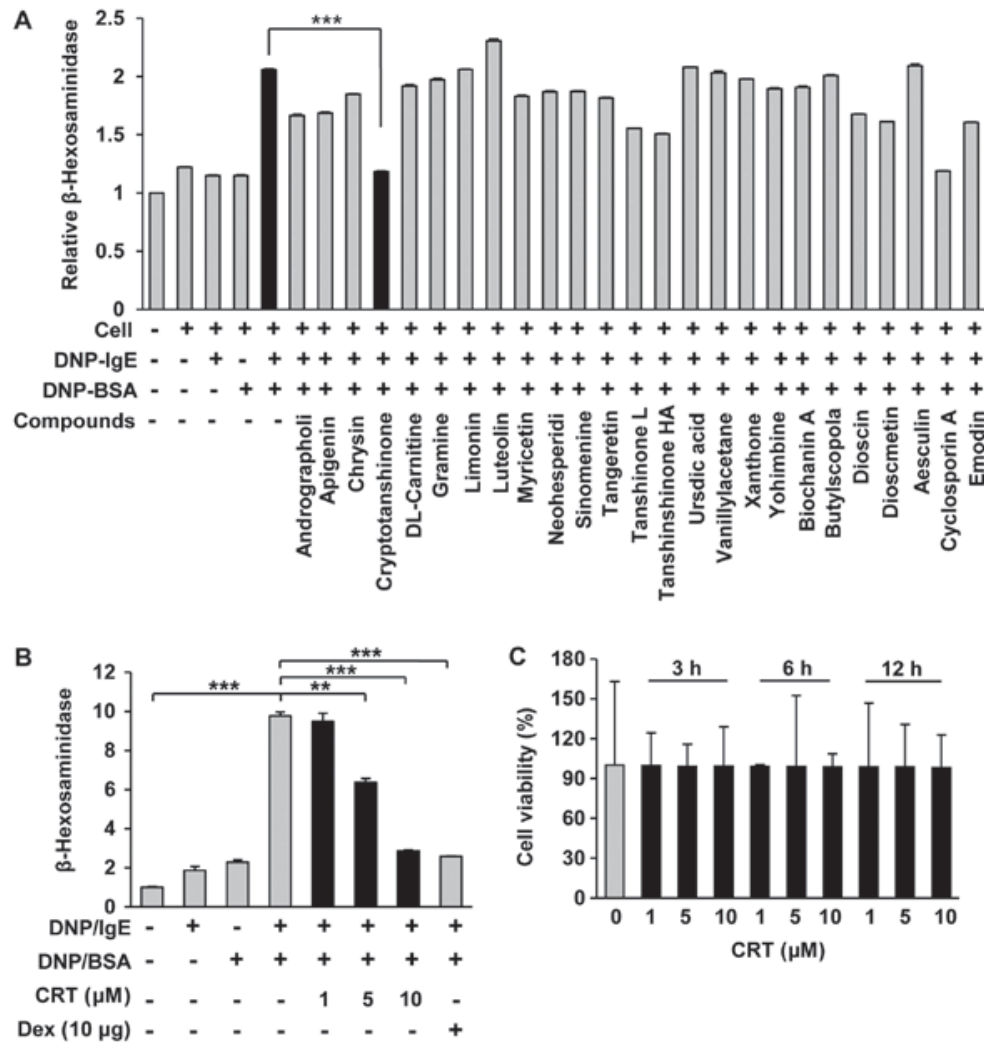

Figure 1. CRT suppresses degranulation in RBL-2H3 cells. (A) Screening of natural compounds by DNP/IgE and DNP/BSA stimulation in mast cells. The amount of degranulation was determined by measuring $\beta$-hexosaminidase levels. (B) RBL-2H3 cells were sensitised with anti-DNP-IgE for $16 \mathrm{~h}$ followed by treatment with various concentrations of CRT $1 \mathrm{~h}$ before DNP/BSA stimulation. The measurement of $\beta$-hexosaminidase release was performed after an additional $1 \mathrm{~h}$ incubation. Dex was used as the positive control. (C) RBL-2H3 cells were treated as (B) and then cell numbers were counted at the indicated additional incubation time using a haemocytometer after trypan blue staining. Data are expressed as the mean \pm standard deviation $(\mathrm{n}=3){ }^{* * *} \mathrm{P}<0.01{ }^{* * * *} \mathrm{P}<0.001$. IgE, immunoglobulin E; DNP/IgE, anti-dinitrophenyl IgE isotype; BSA, bovine serum albumin; CRT, cryptotanshinone.
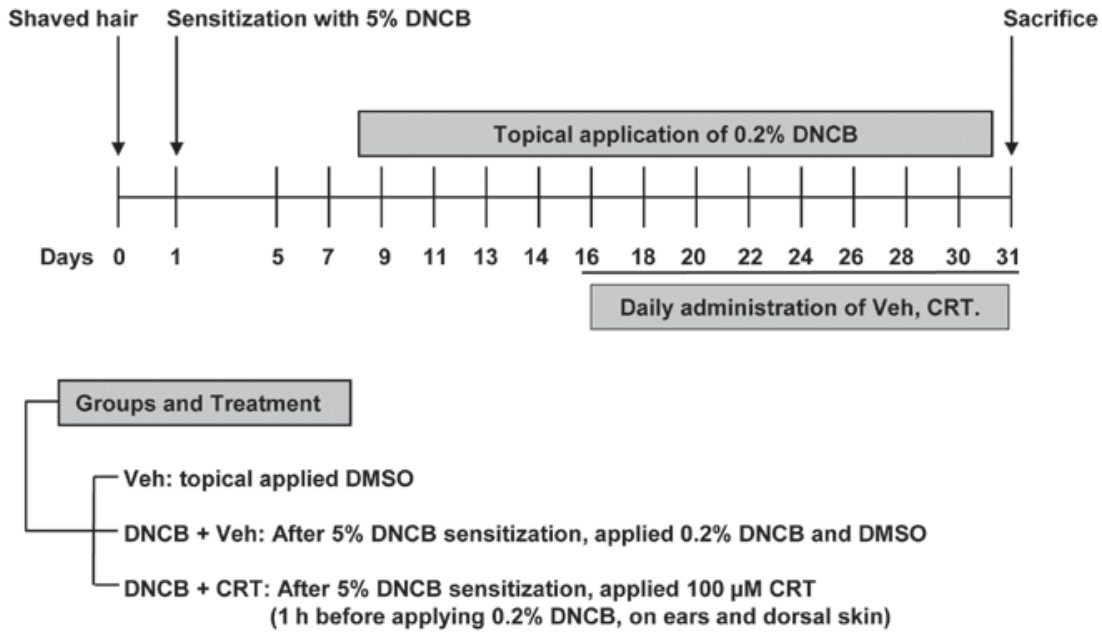

Figure 2. Schematic diagram of developing DNCB-induced atopic dermatitis-like dermatitis inflammation. For animal experiments, 6-week-old Balb/c mice were divided into three groups. DMSO was administered to the Veh group $(\mathrm{n}=5)$. The control group $(\mathrm{DNCB}+\mathrm{Veh} ; \mathrm{n}=5)$ was sensitized with $5 \%$ DNCB on the first day of the experiment. After 5 days later, sensitized mice were subsequently challenged with $0.2 \%$ DNCB on the dorsal skin and on the left and right ears on every other day. The experimental group (DNCB + CRT; $\mathrm{n}=5$ ) mice were pre-treated with $100 \mu \mathrm{M}$ of CRT $1 \mathrm{~h}$ prior to every DNCB challenge. Veh, vehicle; DNCB, 1-chloro-2,4-dinitrobenzene; CRT, cryptotanshinone; DMSO, dimethyl sulfoxide.

induced by DNCB (Fig. 3C). Next, immunofluorescence analysis was performed to investigate whether the effects of mitigation of ear swelling induced by CRT could be associated with a reduction in immune cell recruitment to the inflammatory skin lesion. Interestingly, CRT treatment also restored
DNCB-induced excessive accumulation of CD11b-positive immune cells in the ear skin lesion (Fig. 3D), indicating that CRT alleviates DNCB-induced AD-like skin symptoms. In addition, CRT greatly suppressed increased spleen weights by DNCB (Fig. 3E). Given that the spleen weight is an 


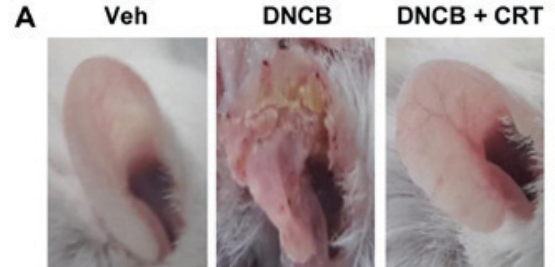

C
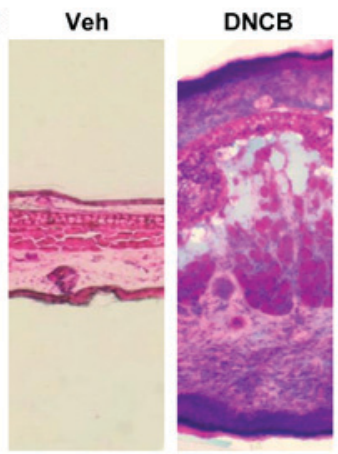

DNCB + CRT
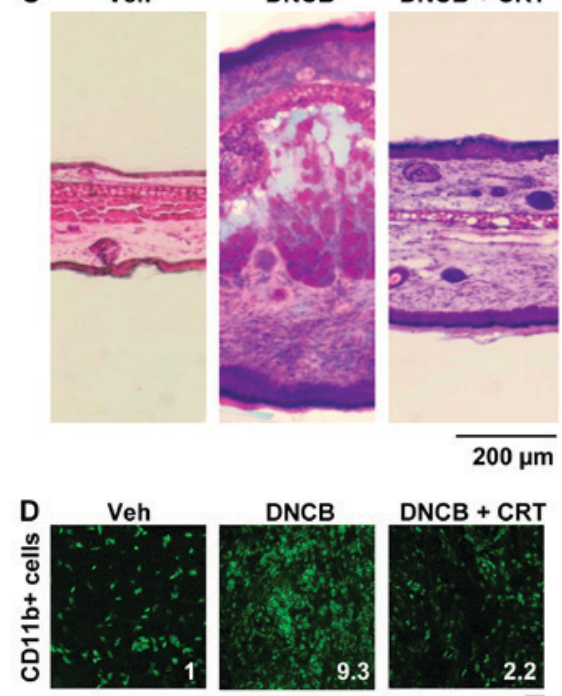

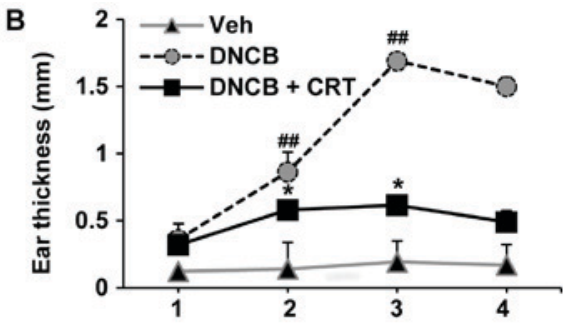

E

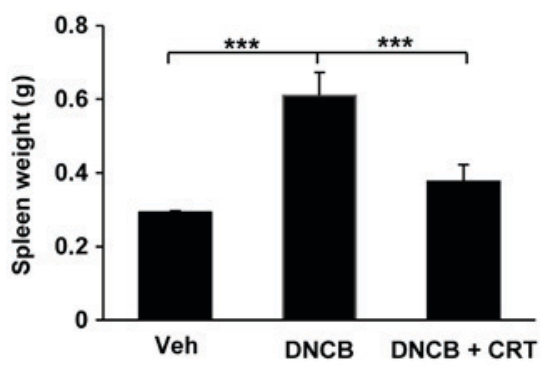

Figure 3. CRT alleviates the DNCB-induced atopic dermatitis. (A) Topical application of DNCB on the shaved dorsal skin and right and left ears was performed according to the schematic protocol in Fig. 2 and $100 \mu \mathrm{M}$ CRT was applied $1 \mathrm{~h}$ prior to each DNCB challenge. Images were captured on the final day of the experiment. (B) Ear swelling was evaluated three times using a dial caliper at the indicated week and then graphed. ${ }^{\# \#} \mathrm{P}<0.01 \mathrm{vs}$. Veh and ${ }^{*} \mathrm{P}<0.05$ vs. DNCB at the same time points. (C) Ear specimens were prepared and stained using haematoxylin and eosin. (D) Tissue sections were stained with anti-CD11b antibody and then captured using a microscope. (E) Spleens were weighed. Data are expressed as mean \pm standard deviation $(\mathrm{n}=5)$. ${ }^{* * *} \mathrm{P}<0.001$. Veh, vehicle; DNCB, 1-chloro-2,4-dinitrobenzene; CRT, cryptotanshinone; CD11b, cluster of differentiation molecule 11b.

indicator evaluating the degree of inflammation, those observations suggest that CRT exerts the anti-AD effect through its anti-inflammatory effect.

CRT decreased transcription levels of pro-inflammatory cytokines and inhibited the $N F-\kappa B$ signalling pathway in the AD-like skin lesion in the DNCB-induced animal model. Since the allergen-specific IgE involves in the initial phase of the allergic response, the increased levels of allergen-specific IgE is a hallmark of AD. Thus, we isolated serum and ear tissue lysates and then measured IgE levels by ELISA. DNCB-challenged mice exhibited a significant increase in serum IgE levels, but no significant changes were observed with CRT treatment compared to DNCB-challenged mice (Fig. 4A). However, IgE levels in lysates from ear tissues were decreased by CRT treatment (Fig. 4B), implying that CRT decreases the local IgE level at nearby inflamed region. Next, we evaluated whether the CRT has inhibitory effects on pro-inflammatory cytokines including IL-1 $\beta$ and TNF- $\alpha$, which are known to be upregulated and play important roles associated with the NF- $\kappa \mathrm{B}$ signalling pathway under inflammation-challenged conditions. Therefore, the transcription levels of pro-inflammatory cytokines in ear tissues were determined by qRT-PCR. As expected, CRT treatment significantly reduced DNCB-challenged upregulation of IL-1 $\beta$ (Fig. 4C) and TNF- $\alpha$ (Fig. 4D). Next, we examined the inhibitory effect of CRT on the activation of the NF- $\mathrm{B}$ pathway in inflamed ear tissues isolated from a DNCB-induced AD animal model. CRT showed a suppressive effect on DNCB-induced NF- $\kappa$ B p65 phosphorylation (Fig. 4E).

CRT inhibited the transcriptions of inflammatory cytokines by suppression of IgE-mediated ERK $1 / 2$ and $N F-\kappa B$ activation in RBL-2H3 cells. Cross-linking of the IgE/antigen complex stimulates mast cells to produce pro-inflammatory cytokines through activation of the MAPK ERK $1 / 2$ and NF- $\kappa \mathrm{B}$ signalling pathways. Thus, the effect of CRT on ERK $1 / 2$ and NF- $\kappa \mathrm{B}$ signalling pathway activation was examined using RBL-2H3 cells. IgE-cross-linking by DNP/BSA treatment activated EKR $1 / 2$ by increasing phosphorylation, but pre-treatment with CRT dose-dependently suppressed ERK $1 / 2$ activation in RBL-2H3

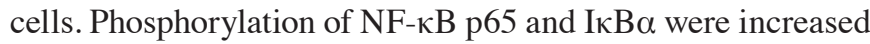
by IgE cross-linking; however, CRT treatment inhibited their phosphorylations in a dose-dependent manner (Fig. 5A).

Next, we asked whether CRT directly regulates the activity of NF- $\kappa$ B. To address this, 293T cells were transfected with $\mathrm{NF}-\kappa \mathrm{B}$ luciferase reporter gene, because $293 \mathrm{~T}$ cells show higher transient transfection efficiency than RBL-2H3 cells. The transfected cells were treated with LPS treatment to activate NF- $\kappa$ B. Following co-treatment with CRT and LPS, CRT significantly suppressed LPS-activated NF- $\kappa$ B luciferase activity (Fig. 5B). These results indicated that CRT significantly 

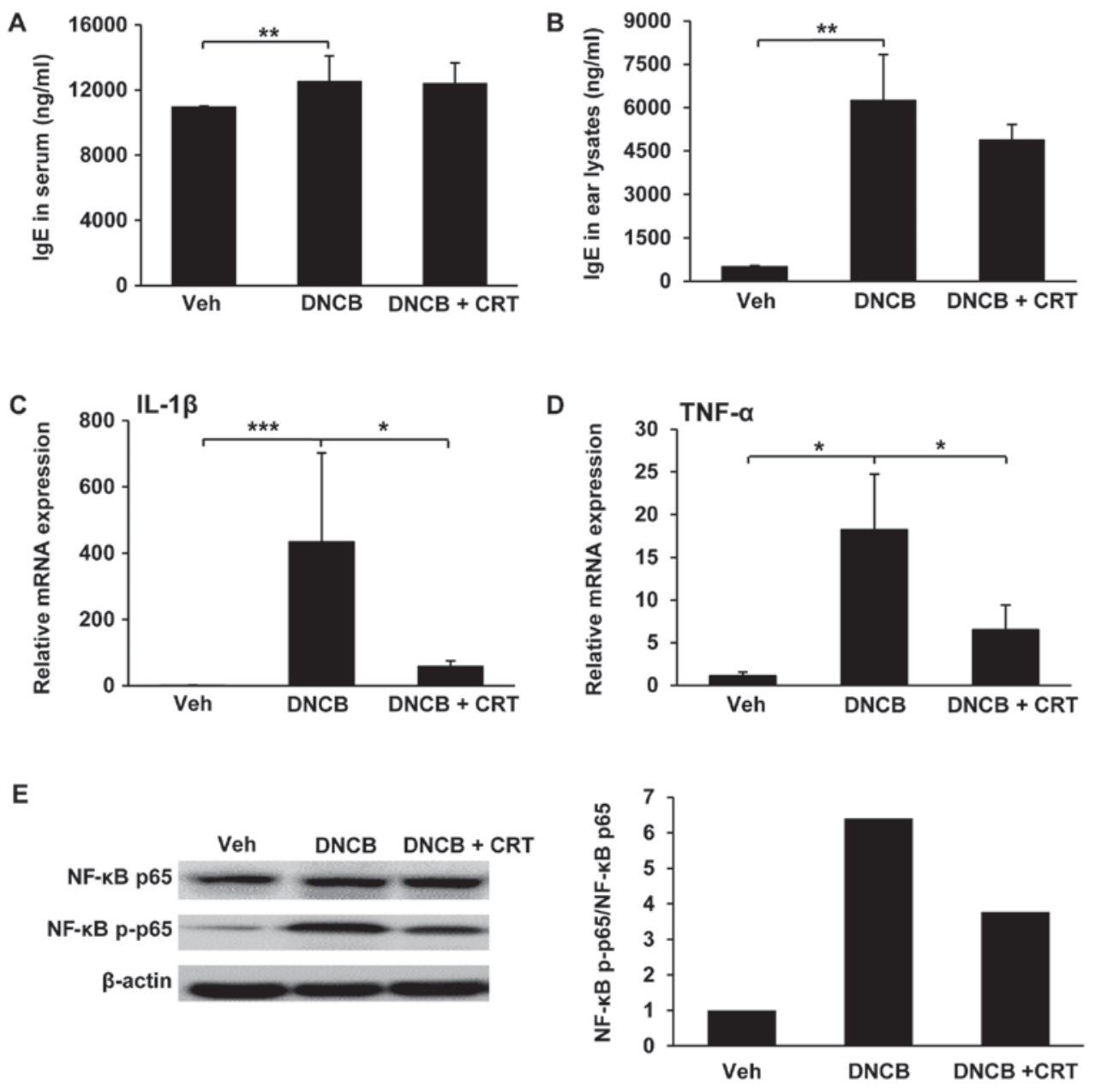

Figure 4. CRT decreases DNCB-upregulated pro-inflammatory cytokines and IgE in atopic dermatitis skin lesions. IgE levels in (A) serum and (B) lysates from ear tissue were evaluated by ELISA. Total RNA was isolated from ear tissues and transcriptional expression levels of pro-inflammatory cytokines (C) IL-1 $\beta$ and (D) TNF- $\alpha$ were determined by reverse transcription-quantitative polymerase chain reaction. Results are expressed as the mean \pm standard deviation ( $\mathrm{n}=3$ ).

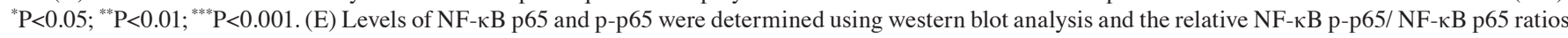
were quantified. Veh, vehicle; DNCB, 1-chloro-2,4-dinitrobenzene; CRT, cryptotanshinone; IL, interleukin; TNF, tumor necrosis factor; NF, nuclear factor; p-, phosphorylated; IgE, immunoglobulin E.

mitigates the IgE-mediated NF- $\kappa \mathrm{B}$ activation in RBL-2H3 cells. Moreover, CRT treatment also decreased the transcription levels of TNF- $\alpha$ and IL-6 increased by IgE cross-linking in RBL-2H3 cells (Fig. 5C). In addition to these inflammatory cytokines, the expression of MCP-1, a key chemokine involved in the stimulation of infiltration and migration of leukocytes towards the inflammatory lesion, was also examined. Interestingly, the IgE cross-linking-induced upregulation of MCP-1 was completely restored to normal levels by CRT treatment (Fig. 5C), suggesting that the decreased accumulation of CD11b positive cells in AD-like skin lesions from CRT-treated mice might be caused by a reduced expression of chemoattractant MCP-1.

CRT inhibited IgE-mediated mast cell activation through suppression of Lyn/Syk phosphorylation and its downstream signalling pathway. Next, to reveal exact molecular targets of CRT, we examined effect of CRT on phosphorylation of Lyn and Syk kinases, which are the most upstream kinases responsible for mast cell activation. CRT treatment suppressed phosphorylation levels of Lyn and Syk in activated RBL-2H3 cells (Fig. 6A). We also examined phosphorylation levels of PLC $\gamma, \operatorname{PKC} \delta$, and IKK $\beta$, which are downstream target molecules of p-Lyn and p-Syk. CRT also greatly suppressed phosphorylation of PLC $\gamma$, PKC, and IKK $\beta$ in a dose-dependent manner (Fig. 6B). CRT effects on p-PLC $\gamma$, p-PKC, and p-IKK $\beta$ showed its best efficiency with $10 \mu \mathrm{M}$ of concentration. These results suggest that CRT suppresses IgE-mediated mast cell degranulation by inhibiting the activation of Lyn and Syk kinases (Fig. 6C).

\section{Discussion}

Chronic $\mathrm{AD}$ patients have a higher risk of developing allergic rhinitis and asthma, which are triggered by pro-inflammatory mediators released from activated and infiltrated immune cells including mast cells, neutrophils, and macrophages into skin lesions $(20,21)$. To prevent complications and relieve AD symptoms, powerful immunosuppressive steroids are used for treatment, but the beneficial effects are short-lived; thus, patients have to take these steroid drugs chronically. Furthermore, taking steroid drugs continuously for prolonged periods of time leads to severe side effects such as blood disorders, irregular heartbeat, and psychological interference (22). Accordingly, 

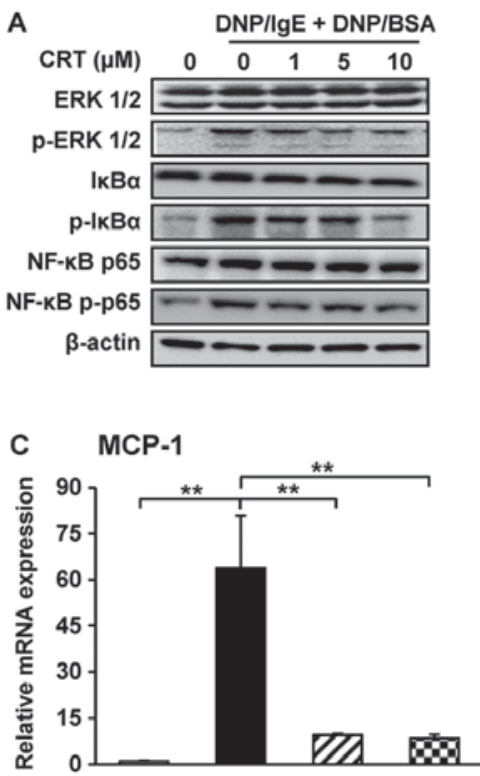
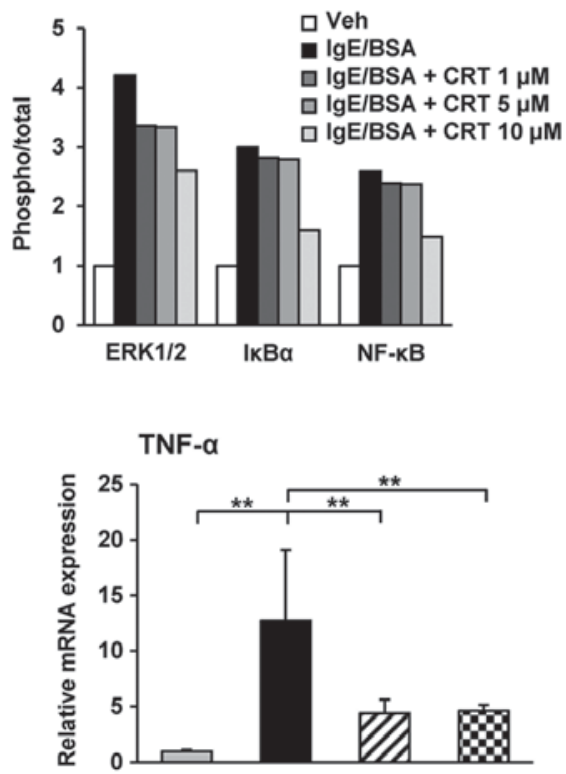
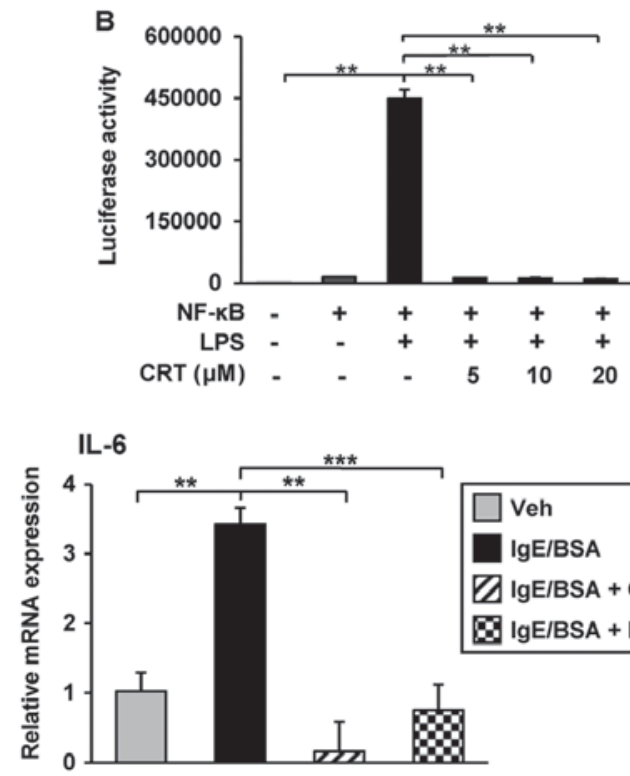

Figure 5. CRT downregulates pro-inflammatory cytokines by inhibiting the IgE/antigen complex-induced extracellular signal regulated kinase $1 / 2$ and NF-кB signalling pathways. (A) RBL-2H3 cells were sensitised with anti-DNP/IgE for $16 \mathrm{~h}$ followed by treatment with various concentrations of CRT $30 \mathrm{~min}$ prior to

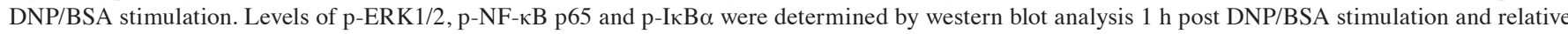

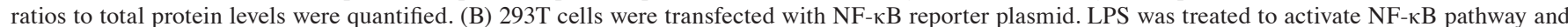
then its activity was evaluated using luciferase assay kit. (C) RBL-2H3 cells were sensitised with anti-DNP-IgE for $16 \mathrm{~h}$ followed by treatment with $10 \mu \mathrm{M}$ CRT $30 \mathrm{~min}$ before DNP/BSA stimulation. Total RNA was isolated $1 \mathrm{~h}$ after DNP/BSA stimulation and mRNA levels of MCP-1, TNF- $\alpha$ and IL-6 were determined by reverse transcription-quantitative polymerase chain reaction. Dex was used as the positive control. Experiments were performed at triplicates three times and representative data are presented as the mean \pm standard deviation. ${ }^{* *} \mathrm{P}<0.01 ;{ }^{* * *} \mathrm{P}<0.001$. CRT, cryptotanshinone; IL, interleukin; TNF, tumor necrosis factor; NF, nuclear factor; p-, phosphorylated; IgE, immunoglobulin E; MCP, monocyte chemoattractant protein 1; DNP/IgE, anti-dinitrophenyl IgE isotype;

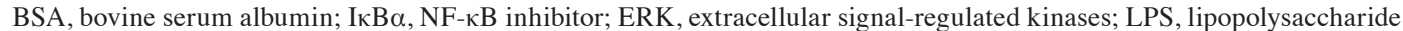

identifying biologically active natural compounds from medicinal plants and developing alternative anti-AD drugs with fewer side effects are in demand to alleviate AD.

In this study, we evaluated the anti-AD effects of CRT using a DNCB-induced AD mouse model, which is typically used for studying the pathogenesis of $\mathrm{AD}$ (4). Topical application of CRT attenuated ear swelling induced by DNCB. In addition, the excessive accumulation of CD11b-positive immune cells in skin lesions triggered by repeated exposure to DNCB on Balb/c mice was dramatically decreased by CRT treatment. In addition, DNCB is known to significantly increase TNF- $\alpha$ and IL-1 $\beta$ levels in mice and these cytokines enhance the expression of adhesion molecules and increase vascular permeability and facilitating the recruitment of inflammatory cells to the skin lesion (23-26). In our study, CRT strongly suppressed mRNA levels of DNCB-induced TNF- $\alpha$ and IL-1 $\beta$, likely resulting in the observed blocked recruitment of immune cells to the skin lesion. However, further studies are required to identify immune cell types decreased by CRT, because monocytes, neutrophils, basophils, mast cells, and eosinophils all express CD11b on their cell surfaces. Conversely, it has previously been reported that extracts from Salvia miltiorrhiza Bunge show immunomodulatory effects by increasing the population of host immune cells, including macrophages, natural killer (NK) cells, and peripheral lymphocytes, and by decrease in serum levels of IgE and the pro-inflammatory cytokine IL-1 $\beta$ against Listeria monocytogenes infection in $\mathrm{Balb} / \mathrm{c}$ mice (27). Thus, CRT could be the major component in Salvia miltiorrhiza Bunge extracts which exerts the potent anti-AD effects.
We showed that CRT exerts anti-AD effect through inhibition of the mast cell degranulation in mast cells. Upon IgE/antigen stimulation, the immunoreceptor tyrosine-based activation motif (ITAM) region of FceRI receptor which is on the mast cell surface is phosphorylated and the initial signalling protein kinases Lyn and Syk are recruited to the ITAM. Then, Lyn and Syk are activated through autophosphorylation, which leads to phosphorylation of the transmembrane adaptor linker for activation of T cells (LAT). Phosphorylated LAT which is a scaffold for multimolecular signalling complexes and activates PLC $\gamma$ through phosphorylation. The activated PLC $\gamma$ hydrolyses phosphatidylinositol biphosphate ( $\mathrm{PIP}_{2}$ ) to generate second signalling molecules $\mathrm{IP}_{3}$ and $\mathrm{DAG}$, which activate PKC $\delta$ through phosphorylation. Then, activated PKC $\delta$ phosphorylates IKK $\beta$ so IKK $\beta$ moves to the plasma membrane, resulting in the induction of mast cell degranulation $(6,11,28,29)$. In this study, novel function of CRT on phosphorylations of Lyn/Syk kinases in mast cells is elucidated for the first time. Furthermore, it is likely that this inhibitory effect of CRT on Lyn/Syk kinases negatively affected activities of their downstream signalling molecules including PLC $\gamma$, PKC $\delta$, and IKK $\beta$, which leads to decrease in mast cell degranulation by CRT treatment.

Besides the inhibitory effect of CRT on mast cell degranulation, here we provide additional evidence that CRT exerts anti-AD effects through inactivation of MAPK and NF- $\kappa B$. It has been reported that CRT regulates the activities of MAPK and NF- $\mathrm{BB}$ in various cell types. In rhabdomyosarcoma, hepatoma, and breast carcinoma, CRT activates MAPK p38/JNK and suppresses ERK1/2, followed 


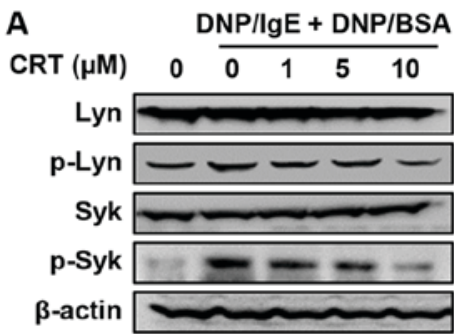

B

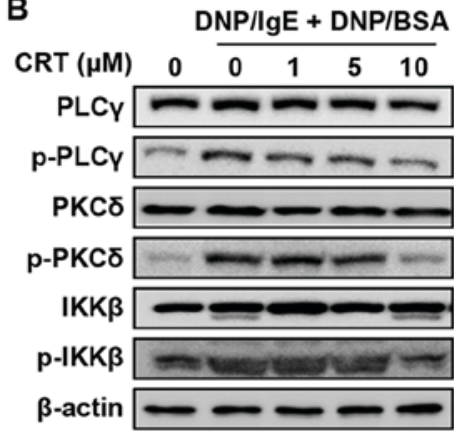

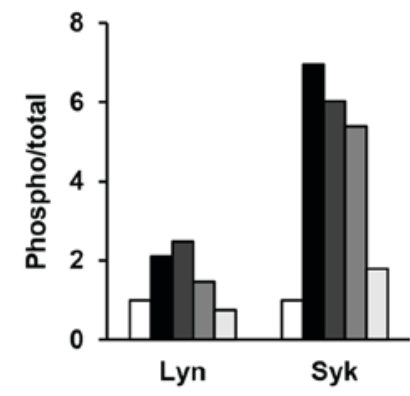

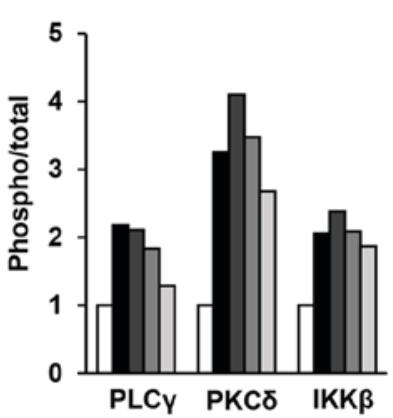

C

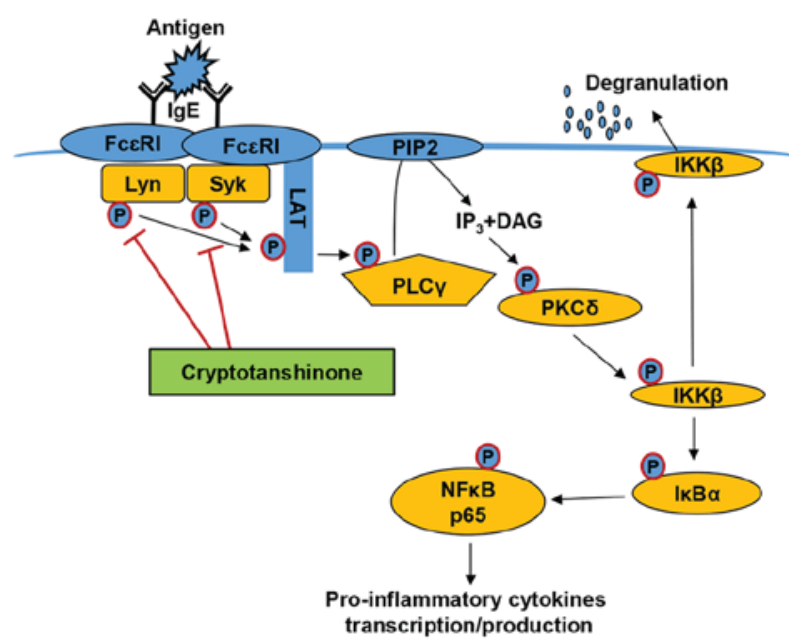

$\square$ Veh $\square \operatorname{lgE} / B S A \quad \square \lg E / B S A+C R T 1 \mu M \quad \square \operatorname{lgE} / B S A+C R T 5 \mu M \quad \square \lg E / B S A+C R T 10 \mu M$

Figure 6. CRT inhibits the signalling pathways of Lyn and Syk (A and B) RBL-2H3 cells were sensitised with anti-DNP-IgE for 16 h followed by treatment with various concentrations of CRT 30 min before DNP/BSA stimulation. Levels of phosphorylated (A) Lyn and Syk, and (B) PLC $\gamma$, PKC $\delta$ and IKK $\beta$ were determined by western blot analysis $1 \mathrm{~h}$ after DNP/BSA stimulation. The relative ratio of phosphorylated protein to total protein levels were quantified. (C) Schematic diagram indicates how CRT suppresses mast cell degranulation. CRT, cryptotanshinone; DNP/IgE, anti-dinitrophenyl IgE isotype; DNA/BSA, dinitrophenyl-bovine serum albumin; PLC $\gamma$, phospholipase $\mathrm{C} \gamma$; PKC, phospho-protein kinase C; IKK, IкB kinase; IgE, immunoglobulin E; Veh, vehicle; Syk, spleen tyrosine kinase.

by caspase-independent apoptosis $(10,30,31)$. In chronic myeloid leukaemia cells, CRT enhances TNF- $\alpha$-induced apoptosis through the activation of MAPK p38 (32). In smooth muscle cells, CRT exerts anti-migration/invasion effect as it inhibits TNF- $\alpha / N F-\kappa B$ signalling pathway (33). In this study, we elucidated the anti-inflammatory role of CRT in mast cells as CRT suppresses the IgE/antigen-induced phosphorylation of ERK $1 / 2$ and $\mathrm{I} \kappa \mathrm{B} \alpha / \mathrm{NF}-\kappa \mathrm{B}$. Furthermore, the luciferase assay revealed that CRT directly inhibits the LPS-induced NF- $\kappa \mathrm{B}$ activity, suggesting that CRT decreases the transcriptions of pro-inflammatory cytokines by downregulation of $N F-\kappa B$ activity. Given that IKK $\beta$ regulates $I \kappa B \alpha / N F-\kappa B$ signalling pathway and that the activity of IKK $\beta$ is controlled by the Lyn/Syk signalling pathway, the suppressive effect of CRT on NF- $\kappa \mathrm{B}$ activity is also considered to be a downstream effect of CRT-induced inhibition of the Lyn/Syk signalling pathway as well as degranulation. Nonetheless, the limitation of this study is that the $N F-\kappa B$ activity was not measured in mast cells because of low transfection efficiency.

In conclusion, we provide evidence that CRT could be developed as an anti-AD drug because it targets Lyn/Syk kinases which are the most upstream signalling molecules for mast cell degranulation and the production of inflammatory cytokines (Fig. 6C). Further studies examining whether CRT can directly inhibit autophosphorylations of Lyn/Syk kinases and suppress the recruitment of Lyn/Syk kinases to ITAM of FceRI will be more valuable for therapeutic drug development using CRT.

\section{Acknowledgements}

Not applicable.

\section{Funding}

The present study was supported by the National Research Foundation of Korea grant funded by the Korean government (grant nos. NRF-2016R1A2B2011683, 2016R1A5A1011974, NRF-2015M3A9B6027818 and NRF-2016R1A6A3A11931083).

\section{Availability of data and materials}

The datasets used and/or analyzed during the current study are available from the corresponding author on reasonable request.

\section{Authors' contributions}

SB and SH designed the study, performed the experiments, interpreted the data and were major contributors in writing the manuscript. SL setup the degranulation assay using the RBL-2H3 cell line. ALJ interpreted the results. HIK, JYP and $\mathrm{AB}$ performed the RT-PCR and western blot analysis. J-SL setup of the DNCB-induced AD mouse model. M-SL contributed to the preliminary screening of 133 natural compounds. YY conceived the project and interpreted the data. All authors read and approved the final manuscript. All authors read and approved the final manuscript. 


\section{Ethics approval and consent to participate}

The present study was approved by the Institutional Animal Care and Use Committee of Sookmyung Women's University (approval no. SMWU-IACUC-1611-035).

\section{Consent for publication}

Not applicable.

\section{Competing interests}

The authors declare that they have no competing interests.

\section{References}

1. Park S, Lee JB and Kang S: Topical application of Chrysanthemum indicum L. attenuates the development of atopic dermatitis-like skin lesions by suppressing serum IgE levels, IFN- $\gamma$, and IL-4 in Nc/Nga mice. Evid Based Complement Alternat Med 2012: 821967, 2012.

2. Kim SR, Choi HS, Seo HS, Ku JM, Hong SH, Yoo HH, Shin YC and Ko SG: Oral administration of herbal mixture extract inhibits 2,4-dinitrochlobenzene-induced atopic dermatitis in BALB/c mice. Mediators Inflamm 2014: 319438, 2014.

3. Martel BC, Lovato P, Bäumer W and Olivry T: Translational animal models of atopic dermatitis for preclinical studies. Yale J Biol Med 90: 389-402, 2017.

4. Lee KS, Jeong ES, Heo SH, Seo JH, Jeong DG and Choi YK: A novel model for human atopic dermatitis: Application of repeated DNCB patch in BALB/c mice, in comparison with $\mathrm{NC} / \mathrm{Nga}$ mice. Lab Anim Res 26: 95-102, 2010

5. Kim SR, Choi HS, Seo HS, Choi YK, Shin YC and Ko SG: Topical application of herbal mixture extract inhibits ovalbuminor 2,4-dinitrochlorobenzene-induced atopic dermatitis. Evid Based Complement Alternat Med 2012: 545497, 2012

6. Shim J, Kennedy RH, Weatherly LM, Hutchinson LM, Pelletier JH, Hashmi HN, Blais K, Velez A and Gosse JA: Arsenic inhibits mast cell degranulation via suppression of early tyrosine phosphorylation events. J Appl Toxicol 36: 1446-1459, 2016.

7. Suzuki K and Verma IM: Phosphorylation of SNAP-23 by IkappaB kinase 2 regulates mast cell degranulation. Cell 134 485-495, 2008

8. Hepp R, Puri N, Hohenstein AC, Crawford GL, Whiteheart SW and Roche PA: Phosphorylation of SNAP-23 regulates exocytosis from mast cells. J Biol Chem 280: 6610-6620, 2005.

9. Park JH, Lee B, Kim HK, Kim EY, Kim JH, Min JH, Kim S, Sohn Y and Jung HS: Peimine inhibits the production of proinflammatory cytokines through regulation of the phosphorylation of NF- $\kappa \mathrm{B}$ and MAPKs in HMC-1 cells. Pharmacogn Mag 13 (Suppl 2): S359-S364, 2017.

10. Lian Q, Cheng Y, Zhong $C$ and Wang F: Inhibition of the IgE-mediated activation of RBL-2H3 cells by TIPP, a novel thymic immunosuppressive pentapeptide. Int J Mol Sci 16: 2252-2268, 2015.

11. Choi HS and Kim KM: Tanshinones inhibit mast cell degranulation by interfering with $\mathrm{IgE}$ receptor-mediated tyrosine phosphorylation of PLCgamma2 and MAPK. Planta Med 70: 178-180, 2004.

12. Wang BQ: Salvia miltiorrhiza: Chemical and pharmacological review of medicinal plant. J Med Plants Res 4: pp2813-2820, 2010.

13. Gao H, Sun W, Zhao J, Wu X, Lu JJ, Chen X, Xu QM, Khan IA and Yang S: Tanshinones and diethyl blechnics with anti-inflammatory and anti-cancer activities from Salvia miltiorrhiza Bunge (Danshen). Sci Rep 6: 33720, 2016.

14. Liu B, Du Y, Cong L, Jia X and Yang G: Danshen (Salvia miltiorrhiza) compounds improve the biochemical indices of the patients with coronary heart disease. Evid Based Complement Alternat Med 2016: 9781715, 2016.

15. Chen W and Chen G: Danshen (Salvia miltiorrhiza Bunge): A prospective healing sage for cardiovascular diseases. Curr Pharm Des 23: 5125-5135, 2017.
16. Shin DS, Kim HN, Shin KD, Yoon YJ, Kim SJ, Han DC and Kwon BM: Cryptotanshinone inhibits constitutive signal transducer and activator of transcription 3 function through blocking the dimerization in DU145 prostate cancer cells. Cancer Res 69: 193-202, 2009.

17. Passante E and Frankish N: The RBL-2H3 cell line: Its provenance and suitability as a model for the mast cell. Inflamm Res 58: 737-745, 2009.

18. Metzger H, Alcaraz G, Hohman R, Kinet JP, Pribluda V and Quarto R: The receptor with high affinity for immunoglobulin E. Annu Rev Immunol 4: 419-470, 1986.

19. Seldin DC, Adelman S, Austen KF, Stevens RL, Hein A, Caulfield JP and Woodbury RG: Homology of the rat basophilic leukemia cell and the rat mucosal mast cell. Proc Natl Acad Sci USA 82: 3871-3875, 1985 .

20. Bantz SK, Zhu Z and Zheng T: The atopic march: Progression from atopic dermatitis to allergic rhinitis and asthma. J Clin Cell Immunol 5: pii: 202, 2014.

21. Akdis CA, Akdis M, Bieber T, Bindslev-Jensen C, Boguniewicz M, Eigenmann P, Hamid Q, Kapp A, Leung DY, Lipozencic J, et al: Diagnosis and treatment of atopic dermatitis in children and adults: European Academy of Allergology and Clinical immunology/American Academy of Allergy, Asthma and Immunology/PRACTALL Consensus Report. J Allergy Clin Immunol 118: 152-169, 2006.

22. Ku JM,Hong SH, Kim HI, SEo HS, Shin YC and Ko SG: Effects of Angelicae dahuricae Radix on 2,4-Dinitrochlorobenzene-induced atopic dermatitis-like skin lesions in mice model. BMC Complement Altern Med 17: 98, 2017.

23. Lawrence T: The nuclear factor NF-kappaB pathway in inflammation. Cold Spring Harb Perspect Biol 1: a001651, 2009.

24. Junghans V, Gutgesell C, Jung T and Neumann C: Epidermal cytokines IL-1beta, TNF-alpha, and IL-12 in patients with atopic dermatitis: Response to application of house dust mite antigens. J Invest Dermatol 111: 1184-1188, 1998.

25. Lin G, Gao S, Cheng J, Li Y, Shan L and $\mathrm{Hu} \mathrm{Z:}$ $1 \beta$-hydroxyalantolactone, a sesquiterpene lactone from Inula japonica, attenuates atopic dermatitis-like skin lesions induced by 2,4-dinitrochlorobenzene in the mouse. Pharm Biol 54: 516-522, 2016.

26. Pokharel YR, Lim SC, Kim SC, Heo TH, Choi HK and Kang HK: Sopungyangjae-tang inhibits development of dermatitis in Nc/Nga mice. Evid Based Complement Alternat Med 5: 173-180, 2008.

27. Gao D, Mendoza A, Lu S and Lawrence DA: Immunomodulatory effects of Danshen (Salvia miltiorrhiza) in BALB/c mice. ISRN Inflamm 2012: 954032, 2012.

28. Stone KD, Prussin C and Metcalfe DD: IgE, mast cells, basophils, and eosinophils. J Allergy Clin Immunol 125 (2 Suppl 2): S73-S80, 2010.

29. Kawakami Y, Kitaura J, Satterthwaite AB, Kato RM, Asai K, Hartman SE, Maeda-Yamamoto M, Lowell CA, Rawlings DJ, Witte ON and Kawakami T: Redundant and opposing functions of two tyrosine kinases, Btk and Lyn, in mast cell activation. J Immunol 165: 1210-1219, 2000

30. Lee M, Lee NY, Chung KS, Cheon SY, Lee KT and An HJ: Roxatidine attenuates mast cell-mediated allergic inflammation via inhibition of NF-кB and p38 MAPK activation. Sci Rep 7: 41721, 2017.

31. Chen W, Lui L, Luo Y, Odaka Y, Awate S, Zhou H, Shen T, Zheng S, Lu Y and Huang S: Cryptotanshinone activates p38/JNK and inhibits Erk1/2 leading to caspase-independent cell death in tumor cells. Cancer Prev Res (Phila) 5: 778-787, 2012.

32. Kim JH, Jeong SJ, Kwon TR, Yun SM, Jung JH, Kim M, Lee HJ, Lee MH, Ko SG, Chen CY and Kim SH: Cryptotanshinone enhances TNF- $\alpha$-induced apoptosis in chronic myeloid leukemia KBM-5 cells. Apoptosis 16: 696-707, 2011.

33. Suh SJ, Jin UH, Choi HJ, Chang HW, Son JK, Lee SH, Jeon SJ, Son KH, Chang YC, Lee YC and Kim CH: Cryptotanshinone from Salvia miltiorrhiza BUNGE has an inhibitory effect on TNF-alpha-induced matrix metalloproteinase-9 production and HASMC migration via down-regulated NF-kappaB and AP-1. Biochem Pharmacol 72: 1680-1689, 2006. 